\title{
Perceptions among primary caregivers about the etiology of delirium: a study from a tertiary care centre in India
}

\author{
S Grover, R Shah
}

Department of Psychiatry, Postgraduate Institute of Medical Education and Research, Chandigarh, India

\begin{abstract}
Objective: To study caregivers' perceptions about the cause of delirium and their distress caused by symptoms of delirium Method: Adult caregivers of patients with delirium, who gave consent, were asked about their perceptions of the cause of delirium. Patients were assessed for delirium by using the delirium rating scale-revised version (DRS-R-98). Results: The study included 72 primary caregivers of patients with delirium. About one-third of the caregivers (36.11\%) attributed the symptoms of delirium to non-organic causes like supernatural beliefs, emotional stress resulting from physical illness or various social factors, attention seeking behaviour, or a result of religious disobedience. Approximately eight percent of the caregivers couldn't give any reason for the altered mental state of the patient. Others attributed the symptoms to medical-surgical causes. Conclusion: A significant proportion of caregivers attribute delirium to non-organic causes in this context.
\end{abstract}

Key words: Delirium; Distress; Caregivers; Causality

Received: 24-01-2011

Accepted: $24-06-2011$

doi: http://dx.doi.org/10.4314/ajpsy.v15i3.26

\section{Introduction}

Delirium is a neuropsychiatric disorder characterized by disturbances of consciousness, attention, cognition, thought and perception. ${ }^{1,2}$ These disturbances manifest as abnormal behaviour, delusions, hallucinations, misperception and misrecognition. When a medically ill patient develops these mental symptoms (especially misrecognition, delusions and hallucinations) which are associated with stigma, caregivers often seek alternate modalities of treatment and cure, which at times may be harmful for the patient.

In India, family forms the major support in caring for ill relatives. In almost all situations, when a patient suffering from any illness is admitted into the hospital, relatives stay with the patient for taking care of their day to day needs and medications. Because of poor resources, the caregivers also provide the basic nursing care. Hence, it is important to understand the caregivers' understanding or beliefs of the cause of delirium and also their level of distress when taking care of these patients.

The limited number of published studies from India have focused on the prevalence of delirium in psychiatric referrals or specialized groups and one study described the phenomenology of delirium in children and adolescents. ${ }^{3-7}$ These studies provide

\section{Correspondence}

Dr S Grover

Department of Psychiatry, Postgraduate Institute of Medical Education \&

Research, Chandigarh 160012, India

email: drsandeepg2002@yahoo.com some evidence that delirium is one of the most common diagnostic categories of psychiatric referrals in India. We could not find any study which has evaluated the caregiver's attribution of symptoms to different causes. This provided the impetus for the current study, which investigated the caregivers' perception about the causality of delirium and their distress with symptoms of delirium.

\section{Method \\ Setting}

This study was undertaken at the Post Graduate Institute of Medical Education and Research, Chandigarh, India, a teaching hospital, with various specialist departments, in North India. The Consultation Liaison Services of Department of Psychiatry provides psychiatric cover to all other inpatients in the hospital.

\section{Design}

This study followed a prospective design in which patients were admitted to various medical, surgical and emergency wards and diagnosed with delirium by the consultation liaison services. The second author recruited and assessed all the patients and caregivers according to the research protocol after ethical approval for the study had been obtained from the Research review Committee of Department of Psychiatry, Post Graduate Institute of Medical Education and Research, Chandigarh. Written informed consent was obtained from the primary caregivers and wherever possible, proxy verbal consent was obtained from the patient at the baseline assessment. Patients that met DSM IV 
diagnostic criteria for delirium were included in the study. ${ }^{8}$ The sociodemographic and clinical profiles were recorded in a structured format. A baseline rating of phenomenology and severity of delirium was done by using the revised version of the Delirium rating scale $-98^{9}$ based on the information provided by the patients, caregivers, medical staff and medical records. Subjects with a history of dementia and any other DSM IV axis I psychiatric disorders (except substance use disorders) were excluded. At baseline, the primary caregivers involved in the management of the patient were questioned on their views of the cause of the symptoms of delirium, using an open ended question "What do you think is the reason that the patient has behavioral changes/ is confused?" Responses given by the caregivers were recorded and when more than one cause was given, they were requested to rank causes in order of importance. When more than one caregiver was involved in the care of the patient, they were also invited to participate in the study. Hence, for some patients ratings from more than one caregiver were available. In such cases, the data of more than one caregiver was analysed as independent subjects. All cases diagnosed with delirium between February and June 2010, were included in the study.

\section{Data Analysis}

The data was analyzed by using the Statistical Package for the Social Sciences-version $14 .{ }^{10}$ Descriptive data was analysed as frequencies, percentages, mean and standard deviation.

\section{Results}

The study included 72 caregivers of 53 patients diagnosed with delirium.

\section{Demographic and clinical profile of patients}

The mean age of patients was 45.58 years (SD 19.06), ranging between 18-83 years. The range of years of education varied from 0-17 years (mean $=9.07, \mathrm{SD} 5.31$ ). The majority of patients were males $(\mathrm{N}=38 ; 71.7 \%)$ and of Hindu religion (71\%). The rest were of Sikh religion.

The mean duration of delirium at the time of baseline assessment was 3.88 days (SD 5.02), with a range of 1-30 days. In most of the cases (71.1\%) the delirium was hospital emergent and about two-thirds (62.3\%) of the patients were admitted in the medical wards and nearly one-third (37.7\%) in the surgical wards.

All patients included in the study presented with disturbances of the sleep-wake cycle, impaired orientation and attention, a fluctuating course of symptoms, onset of symptoms associated with emergence of a new medical surgical condition, and the presence of an identifiable underlying physical disorder. Other details of baseline DRS-R98 are shown in Table I. The mean severity score was 23.05 (SD 5.17) and the mean total score was 29.77 (SD 5.35) (Table-1).

\section{Demographic profile of caregivers}

The mean age of the caregivers was 38.76 years (SD 12.23; range 19-72) with the majority being males ( $N=41 ; 57 \%)$. The mean duration of education was 10.76 years (range 0-20 years, SD 5.56). The relationship of caregivers to the patients was: spouses (25\%), children (29.2\%), parents (12.5\%) and other relatives (33.3\%).

Caregivers' perceptions about etiology of symptoms of delirium All the caregivers were asked to describe their understanding of the cause of their relative's current condition, before the

\begin{tabular}{|l|l|l|}
\hline \multicolumn{2}{|l|}{ Table I: Symptom profile of the sample (n=53) } \\
\hline Symptoms (items) & Prevalence & $\begin{array}{l}\text { Score- } \\
\text { mean/ standard } \\
\text { deviation (range) }\end{array}$ \\
\hline Delusions & $11(20.7 \%)$ & $0.4 \pm 0.9(0-3)$ \\
\hline Motor retardation & $18(34 \%)$ & $0.6 \pm 0.9(0-3)$ \\
\hline Thought process abnormality & $42(79.2 \%)$ & $1.6 \pm 1.1(0-3)$ \\
\hline Language abnormalities & $43(81.1 \%)$ & $1.3 \pm 0.8(0-3)$ \\
\hline Perceptual disturbance & $43(81.2 \%)$ & $1.7 \pm 1.1(0-3)$ \\
\hline Labile affect & $44(83 \%)$ & $1.6 \pm 0.9(0-3)$ \\
\hline Visuospatial deficits & $48(93.1 \%)$ & $1.8 \pm 0.8(0-3)$ \\
\hline Impairment of Long-term memory & $49(94.6 \%)$ & $1.6 \pm 0.8(0-3)$ \\
\hline Motor agitation & $50(94.3 \%)$ & $2.3 \pm 0.9(0-3)$ \\
\hline Impairment of Short-term memory & $52(98.6 \%)$ & $2.5 \pm 0.7(0-3)$ \\
\hline Sleep-wake cycle disturbances & $53(100 \%)$ & $2.7 \pm 0.5(2-3)$ \\
\hline Disturbance in Orientation & $53(100 \%)$ & $2.4 \pm 0.6(1-3)$ \\
\hline Disturbance in Attention & $53(100 \%)$ & $2.3 \pm 0.5(1-3)$ \\
\hline $\begin{array}{l}\text { Temporal onset of symptoms } \\
\text { (rate of acuteness of symptoms) }\end{array}$ & $53(100 \%)$ & $3.0 \pm 0.1(2-3)$ \\
\hline Fluctuation of symptoms & $53(100 \%)$ & $1.8 \pm 0.4(1-2)$ \\
\hline Causal attribution to a Physical Disorder & $53(100 \%)$ & $1.9 \pm 0.3(1-2)$ \\
\hline
\end{tabular}

diagnosis was explained to them by the psychiatry consultation-liaison team. Most of the caregivers gave only one cause for the patient's altered mental status and slightly less than half gave 2 etiological reasons and only 1 caregiver gave 3 etiological reasons for the patient's altered mental status as shown in Table II. The various responses could be grouped into certain themes. Of all the caregivers about one-third of them (36.1\%) attributed the symptoms of delirium to nonorganic causes like supernatural causation, mental stress due to physical illness, tension due to social causes, attention seeking, religious disobedience and homesickness (Table II) Another $8.3 \%$ of the caregivers could not give any reason for the altered mental state of the patient. Other etiological causes given by the caregivers are shown in Table II.

\section{Discussion}

Delirium forms the single largest diagnostic category among all the cases seen by Psychiatry consultation-liaison services in our setting. ${ }^{3}$ Hence it is important to understand the beliefs of the caregivers with respect to the cause of symptoms of delirium as it can influence treatment seeking. This study was a preliminary attempt in this direction.

The participants of the present study were predominantly male, with a mean age of about 45 years, who were admitted in the medical wards. This profile of the participants is similar to that of a typical profile of the patient of delirium seen in our setting. ${ }^{3}$

We could not find any study which has evaluated the beliefs about the causation of the symptoms of delirium. In the current study we found that slightly more than one-third of the caregivers attributed the symptoms of delirium to supernatural causes, emotional stress resulting from physical illness or homesickness, tension due to social factors, attention seeking behaviour, or a result of religious disobedience Approximately 


\section{Table II: Perception of caregivers about etiology of symptoms of delirium}

\begin{tabular}{|l|l|l|l|l|}
\hline Etiology & 1 st & 2nd & 3rd & Cumulative \\
\hline Supernatural causation & $4(5.6 \%)$ & $10(13.9 \%)$ & & $6(5.6 \%)$ \\
\hline Stress/tension due to physical illness & $11(15.3 \%)$ & $2(2.8 \%)$ & & $21(15.3 \%)$ \\
\hline Tension due to social stressors & $8(11.1 \%)$ & $1(1.4 \%)$ & & $9(8.3 \%)$ \\
\hline Attention seeking/ personality & $2(2.8 \%)$ & & & $2(2.8 \%)$ \\
\hline Religious disobedience & $1(1.4 \%)$ & & & $1(1.4 \%)$ \\
\hline Home sickness & & $1(1.4 \%)$ & & $1(1.4 \%)$ \\
\hline Unknown & $6(8.3 \%)$ & & & $6(8.3 \%)$ \\
\hline Resulting from medicines/ treatment & $11(15.3 \%)$ & $7(9.7 \%)$ & & $18(15.3 \%)$ \\
\hline Alcohol & $11(15.3 \%)$ & $1(1.4 \%)$ & & $12(15.3 \%)$ \\
\hline Due to physical illness & $6(8.3 \%)$ & $3(4.2 \%)$ & & $9(8.3 \%)$ \\
\hline Due to head injury & $6(8.3 \%)$ & $3(4.2 \%)$ & & $9(8.3 \%)$ \\
\hline Physical weakness & $2(2.8 \%)$ & $2(2.8 \%)$ & & $4(2.8 \%)$ \\
\hline Lack of sleep & $2(2.8 \%)$ & & & $2(2.8 \%)$ \\
\hline Fever & $2(2.8 \%)$ & & & $2(2.8 \%)$ \\
\hline Pain & & $1(1.4 \%)$ & & $1(1.4 \%)$ \\
\hline Due to sensory deficit & & & $1(1.4 \%)$ & $1(1.4 \%)$ \\
\hline
\end{tabular}

\section{Conclusion}

The current study suggests that about one-third of the caregivers attribute the symptoms of delirium to non-organic / 'psychosocial' causes. Considering the overlap in patients or their relatives understanding of the causes of both schizophrenia and delirium, the findings of the present study have important treatment implications. Another important aspect to note is that in the current study the majority of the patients had hospital emergent delirium (patients were admitted for medical reasons and were diagnosed with delirium while in hospital). This could have influenced the reporting of belief about the causation of symptoms. The consultation-liaison psychiatrist and the treating physicians should always explain to the caregivers about the organic etiology of delirium symptoms and its short lasting nature. This can reduce the distress of the caregivers and help them better cope with the crisis and act in an informed manner. The current study was limited by a small sample size, and the fact that we did not use any specific instrument to assess the beliefs about

eight percent of caregivers could not give any reason for the altered mental state of the patient. There is no data to compare the findings of caregivers' views/understanding of the causes of delirium obtained in the present study, but the findings suggest that about half of the caregivers have mistaken beliefs about the causality of delirium. When we view the findings of the current study in the light of beliefs about causes of schizophrenia existing in our community, it is evident that the beliefs about causes of delirium are similar. In one study, Srinivasan \& Thara reported that $12 \%$ of families attributed schizophrenia to supernatural causes and the most common cited cause for schizophrenia was psychosocial stress. ${ }^{11}$ In another study, Charles et al ${ }^{12}$ reported that $54 \%$ of the family members have at least one medically understandable belief about the cause of schizophrenia and the other common causes reported included black magic, punishment by god, evil spirits and destiny. ${ }^{12}$ In the same study it was also reported that because of the belief system, more than half of the caregivers reported that traditional treatments like visiting a temple, traditional healer or a shaman may be beneficial. ${ }^{12}$ Although, the percentage of caregivers reporting the same in the current study is slightly less, there is a considerable overlap. Even though the percentage of caregivers reporting supernatural causes, in the current study, was less, the majority of the caregivers perceived the cause to be of 'psychosocial' origin. These findings have important implications. Although more research would be required to establish further implications, it is understandable that such perceptions may cause impedance to continuation of medical management. This may be more so in our setting where non-medical models of illness are more prevalent. Similarly, although not reported in the literature, it is common practice in medically ill patients to seek the help of religious institutions and traditional healers for their problem. However, in the presence of delirium or psychosis this practice is more common. causes of mental illnesses. Future studies should use structured instruments to assess the etiological models and should focus on the implications of erroneous models on care seeking behaviour.

\section{References}

1. Burns A, Gallagley A, Byrne J. Delirium. J Neurol Neurosurg Psychiatry 2004; 75: 362-367.

2. Gupta N, de Jonghe J, Schieveld J, Leonard M, Meagher D. Delirium phenomenology: What can we learn from the symptoms of delirium? J Psychosom Res 2008; 65: 215-222.

3. Grover S, Subodh BN, Avasthi A, Chakrabarti S, Kumar S, Sharan P, et al. Prevalence and clinical profile of delinium: a study from a tertiary care hospital in north India. Gen Hosp Psychiatry 2009; 31: 25-9.

4. Chaudhury S, Mahar RS, Augustine M. Post-cataractomy delirium a prospective study. Med J Armed Forces India 1991; 47: 286-90.

5. Sood A, Singh P, Gargi PD. Psychiatric morbidity in non-psychiatric geriatric inpatients. Indian J Psychiatry 2006; 48: 56-61.

6. Khurana P, Sharma PSVN, Avasthi A. Prevalence of delirium in geriatric hospitalized general medical population. Indian J Psychiatry 2002; 44: 41-6.

7. Grover S, Malhotra S, Bharadwaj R, Subodh BN, Kumar S. Delirium in children and adolescents: a study from India. Int J Psychiatry Med 2009; 39: 179-87.

8. American Psychiatric Association. Diagnostic and Statistical Manual of Mental disorders. 4th edition - Text Revision. New Delhi, India, Jaypee Brothers Medical Publishers Ltd, 2002, pp 135-180.

9. Trzepacz PT, Mittal D, Torres R, Kanary K, Norton J, Jimerson N. Validation of the Delirium Rating Scale-revised-98: comparison with the Delirium Rating Scale and the cognitive test for delirium. J Neuropsychiatry Clinical Neurosci 2001; 13: 229-241.

10. Statistical Package for Social Sciences (SPSS) version 14.0, for Windows, Chicago: SPSS Inc, 2005.

11. Srinivasan TN, Thara R. Beliefs about causation of schizophrenia: do Indian families believe in supernatural causes? Soc Psychiatry Psychiatr Epidemiol. 2001; 36: 134-40.

12. Charles H, Manoranjitham SD, Jacob KS. Stigma and explanatory models among people with schizophrenia and their relatives in Vellore, south India. Int J Soc Psychiatry. 2007; 53: 325-32. 